$\mathbb{T}$ periodica polytechnica

\author{
Social and Management Sciences \\ $15 / 2(2007) 59$ \\ doi: 10.3311/pp.so.2007-2.03 \\ web: http://www.pp.bme.hu/so \\ (c) Periodica Polytechnica 2007
}

RESEARCH ARTICLE

\section{Al controlled simulation based environmental assessment}

Attila Für

Received 2008-09-14

\begin{abstract}
Environmental assessment as complex assignment usually needs to use substitute economical methodologies to estimate environmental state instead of using direct valuing, since there are no absolute points of references and cross-couplings of causes and impacts are not transparent. In cases, when quantifying and clustering is ambiguous usage of FUZZY set theory can be suggested. AI controlled simulation assists in identifying of unknown cross-couplings, and in determining of relative importances from different points of views. This article highlights a complex simulation based environmental assessment methodology.
\end{abstract}

\section{Keywords}

environmental assessment $\cdot$ modified Leopold matrix $\cdot$ intelligent agents · identification by reconstruction - Knowledge Attributed Petri Nets $\cdot$ sustainable development of regions $\cdot$ transdisciplinary models

\section{Attila Für}

Department of Environmental Economics, BME, H-1111 Budapest, Sztoczek u. 4, Hungary

e-mail: fur.attila@inesys.hu

\section{Introduction}

An alternative simulation based approach of environmental assessment (EA) to the conventional ones is presented and some applications for solving of specific problems are outlined. Usually classical economical environmental assessment is based on determining physical impacts and relationships; valuing impacts in monetary terms; discounting; and risk and uncertainty. Several methodologies had been developed such as economic analysis of projects and policies (e.g. Economic Analysis, Financial Analysis, Social Cost-Benefit Analysis), and others that take environmental costs into consideration. In the more environmentcentric approach there is a possibility to execute assessment process by surrogating market potential, or using expenditures direct valuation values or the willingness-to-pay concept. There are also numerous indirect ways available to give value for environmental elements e.g. Change of Productivity Property Values, Replacement Costs, Loss of Earnings, Wage Differences Shadow Project, Defends Expenditures, Travel Costs, Contingent Valuation, Marketed Goods as Proxies.

As in Fig. 1 delineated the classical EA approach uses an open-loop process to make EA decision and implementation at the end.

As well known, processes of nature and economics are highly complex, and have several cross-couplings, that cannot be determined easily. In the most cases subsystems that should be investigated, are neither transparent nor semi-transparent, so model identification have to be carried out.

Therefore using of dynamic problem solving simulation methodology can be suggested. Beyond the above mentioned problems AI controlled simulation is able to settle iteration process and feedback mechanism that is necessary to be executed after EA decision. As in Fig. 2 shown alternative simulation based EA process have the following main parts:

- General purposes and aims have to be appointed (that can be eventually modified after the $\mathrm{n}^{\text {th }}$ iteration when needed)

- Building adequate model (model of processes, model of measuring, model of evaluating)

- Monitoring model behaviour and reconstruction 


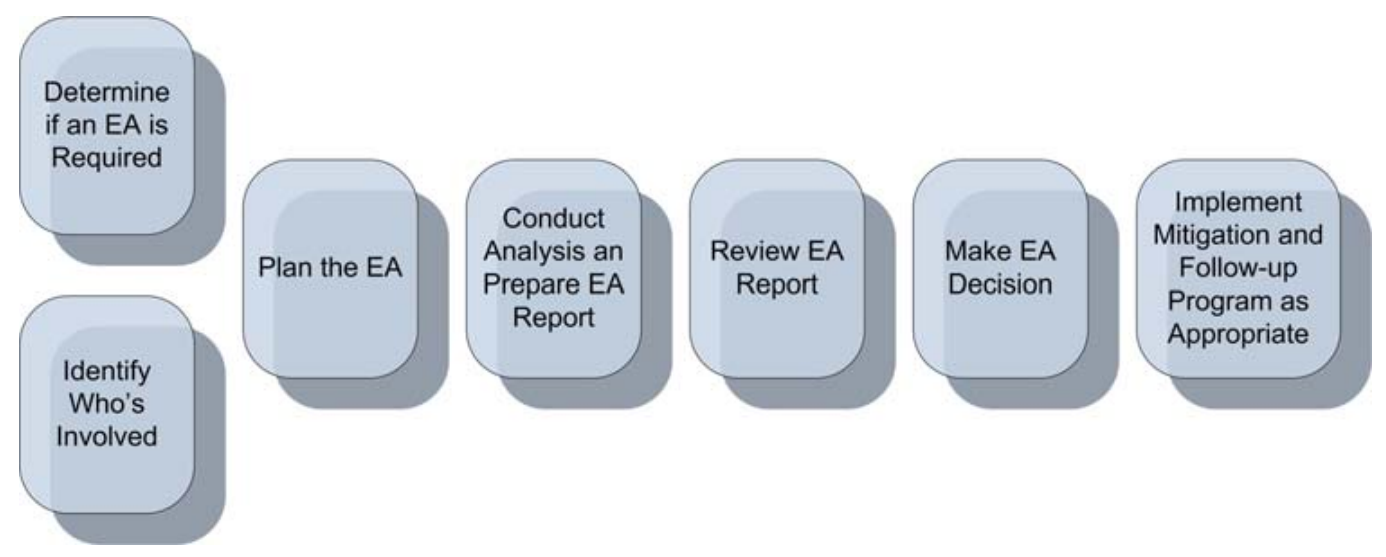

(Source: http://www.ceaa.gc.ca/010/basics_e.htm\#4)

Fig. 1. The classical environmental assessment process

- Making classification, qualifying, clustering, or other assessment

- After decision making, during project realization evaluation is needed to ensure adequate feedback to the whole EA process methodology

\section{Classical EA methods ${ }^{1}$}

\subsection{Change-in-productivity approach}

Change-in-productivity approach uses direct measurement of economical benefits regarding to development in environmental space. For example, a land management project involving soil conservation measures, may yield increased agricultural output. The incremental output can be valued by using standard economic prices [1, 12].

Other estimation of increased or decreased productivity can be recognized by investments on wetlands. In that case fish catch can be projected to market prices, and can be used for evaluating environmental state, or change.

\subsection{Loss-of-earnings approach}

Human health and environmental quality show strong coupling, and are sensitive to transient (fast and significant) changes [2]. Both indicators are hard to measure, but there is a way to use the monetary valuing. In practice economical expenses issue from fall out of production according to damaged health level. This approach uses techniques like valuing earnings that are foregone through premature death, sickness or absenteeism; and increased medical expenditures [12]. The concept of lossof-earnings can be used in the case of road or industrial plant safety, and projects that affect air pollution in major cities.

\subsection{The "Value-of-Health" approach}

As mentioned before, it is highly complicated to assign value to human life (or health of other beings), because this cannot be expressed in monetary units, and probably has infinite value.

\footnotetext{
${ }^{1}$ This chapter is based on $[10]$
}

It is not only question of morality but it is also ponderous on ground of comparability [2].

There are several ways in practice that are taken into consideration as main values of health: e.g. spending on health improvements. Society also places implicitly finite values on human life and health when it makes policy and project decisions that affect environmental quality, workers' health or safety [12].

\subsection{Methods based on surrogate market values}

The methods and techniques described in the following section use market information. As it is not possible to generate and evaluate a virtual environmental market, it is necessary to adapt surrogate market and its prices related to the original value. Each technique has limited applicability, and high level of uncertainty.

\subsubsection{Property value approach}

This kind of valuing is also referred as the hedonic price technique. Usually a value of a property in economical meaning is function of neighbouring environmental state. Change in this state indicates change in monetary value of property. The advantage of this methodology is that properties from the same type can be compared, depending on the surrounding environmental state. Differences in market prices give relevant information about the environment.

The property value approach has been used to analyse the effects of air pollution in certain areas. Where pollution is localized, the method compares prices of houses in affected areas with houses of equal size and similar neighbourhood characteristics elsewhere in the same metropolitan area [12].

\subsubsection{Wage differential approach}

This approach is often questioned on ground of reliability. Theoretically demand for labour in competitive market equals the value of the marginal product and supply of labour varies with working and living conditions in an area. A higher wage is therefore necessary to attract workers to locate in polluted areas or to accept risky work [12]. 


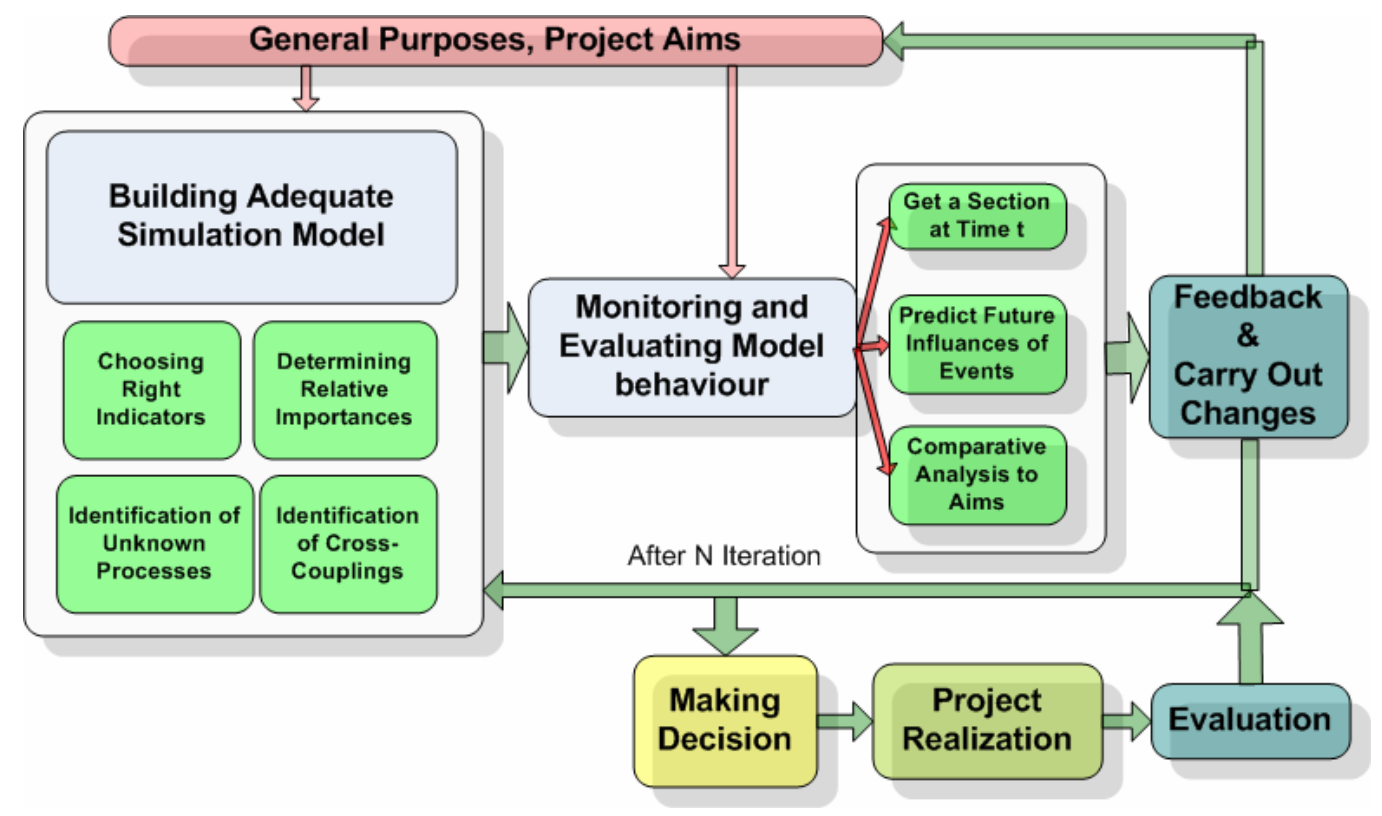

Fig. 2. Simulation aided environmental assessment process

In reality assumptions of theoretical model indicate high level of uncertainty because the approach does not reflect social attributes that may often overshadow environmental aspects.

\subsubsection{Travel cost approach}

This valuing method is usually used in determining economical rate of environmental benefits: e.g. recreational potential of green area can be measured, by assigning the costs of travel paid by visitors - from any distance to the examined location. If rate of visitation and location of origin is known the sum of total costs weighted by the mentioned factors can represent environmental benefits in materialized form [1].

Similarly can be defined travel-time approach that results sometimes more comparable issues.

\subsubsection{Marketed goods as surrogates for non-marketed goods}

In several cases there is a simple way to substitute environmental goods by similar products that are present on market. For example, the value of a non-marketed fish variety can be valued at the price of the most similar fish being sold in local markets [12]. As main disadvantage of this method can be mentioned that it ignores biodiversity.

\subsection{Methods based on potential expenditures or willingness-to-pay}

There are situations, when determining of surrogate market or direct valuing is not possible. In that case the estimation of environmental state can be carried out by a theoretical experiment based on measuring the motivation to pay for recultivation, improvement or keeping environment at constant state.

\subsubsection{Replacement cost approach}

This approach can be applied in the following cases. As preventive method, willingness-to-pay can be measured to avoid damage in environmental quality. By high level of environmental consciousness replacement cost approach results often unduly high costs (or in case of low eco-sensitiveness too low costs), therefore method reliability is uncertain.

In other situation, when damage asset is already present, recultivation costs and costs related to cancelled recultivation can be compared.

\subsubsection{Shadow project approach}

This approach is essentially the same as the replacement cost approach; it is being mentioned increasingly as a way to make operational the concept of sustainability at the project level. This approach is used for evaluating projects with negative environmental impacts involving the design and costing of one or more "shadow projects" that would provide substitute environmental services to compensate for the loss of the original assets [1].

\subsubsection{Contingent valuation method}

This method basically asks people what they are willing to pay for a benefit, and/or what they are willing to accept as compensation for tolerating a cost. This process of "asking" may be carried out either through a direct questionnaire/survey, or by experimental techniques in which subjects respond to various stimuli in "laboratory" conditions [12].

Main disadvantage of this approach is that is based on private preference system that it is function of income level and social or cultural backgrounds. 


\section{Using the simulation methodology}

Although at the beginning of the $21^{s t}$ century the application of simulation to promote problem solving is already a widespread approach; usually it is used in a linear way. This means that a model of the system to be investigated is built, the simulation - under given boundary conditions and external effects - is executed and the behaviour of the system is evaluated. However in a very wide range of fields there is a problem already at the beginning. Namely the model itself is hard to determine and there are only vague ideas about its structure and operation. Among many other cases this is true for social systems, environmental problems, micro- and macro-economy and various systems where in general human behaviour has a significant influence on. In these cases - in order to obtain at least relatively useful and reliable information - the first step should be determining a model corresponding to the system to be investigated with an acceptable fidelity. Consequently before executing the simulation; the determination of the appropriate model is essential. In the approach presented it is intended to solve the above mentioned problem by applying intelligent agents to do the job.

\section{Principles of model identification by reconstruction ${ }^{2}$}

The approach proposed is the following:

- Initial model building based on the information obtained from the experts of the field.

- Run the model using historical data.

- Monitor the model behaviour continuously during dynamic simulation and modify it according to the deviation of model behaviour from that of the real system during the simulation run.

- After the model is determined by the above procedure of identification by reconstruction it can be used to forecast the system behaviour in the future.

In order that the methodology described above should be applicable it is essential that the simulation model should be built in an object oriented way i.e. the model should be built as a network of interconnected and interacting objects [10].

To make this process feasible it is important that the procedure should be automated since if the model reconstruction should be undertaken with human efforts this would require enormous time and energy. Therefore the process - in our proposed solution - is undertaken by intelligent agents (demons) [5] monitoring the behaviour of the simulation model continuously and modifying it [6].

The above mentioned approach is illustrated in Fig. 3 .

The intelligent demons have knowledge bases where the historical data of the model behaviour in the past is stored. The anticipated model operates driven by the historical data provided to its inputs. It delivers its outputs representing the model

\footnotetext{
${ }^{2}$ This chapter is based on $[10$
}

behaviour and the inference engines of the demons compare it with the behavioural information in their knowledge bases, intervene into the model structure and modify it to improve its resemblance to the real system to be investigated. This procedure continues until the initial model is modified to reveal the real system with acceptable fidelity.

The demons can evaluate the information about the model operation after preprocessing it (as e.g. average values, correlation, standard deviation, etc.). The modification of the model can be different as e.g.

- Modification of model element parameters

- Modification of model structure (i.e. changing the structure of the model element network constituting the model as a whole)

- Effects of the interconnected model elements on each other in the simulation model as a whole.

\section{Environmental assessment using identification by reconstruction}

As well known, questions of ecological economics and sustainability, regional environmental policy, protection of nature and identifying the processes of the whole biosphere are the most complicated fields of future environmental strategy that should be investigated by alternative methodologies such as model identification by reconstruction. One of the major areas of the above mentioned is the environmental assessment, that is integrated into all processes of economics and should be taken into consideration not only in the case of feasibility studies for local projects, but also for regional policy and planning sustainable development [1] [2]|[3].

Environmental assessment as qualifying, quantifying, clustering, measuring of unknown or fuzzy defined quantities is a typical problem of identification of soft systems. Assessment - as feedback phenomena for the closed loop control of nature and economy based processes - should be carried out with high precision because of the caused consequences by the interventions.

To highlight the main problem of the classical approach of environmental assessment the notional word has to be analysed. The first part contains the word "environment". What is meant by that composition? The local physical area of living beings? Regional artificial and natural environment? The whole biosphere? It is obvious that each concept is acceptable under given conditions, but using of hybrid aspects are very dangerous, because their model scalability is limited and accuracy of measuring the environmental state will be reduced. Each real system should be approximated by the most adequate model description.

After the object to be investigated has been defined, right indicators have to be selected, that give relevant information on the change of the environmental state. At environmental model synthesis indicators reflect significance, relevant information that is selected and ranked by members of economical and natural aspects considering their points of view on the specific indicators. 


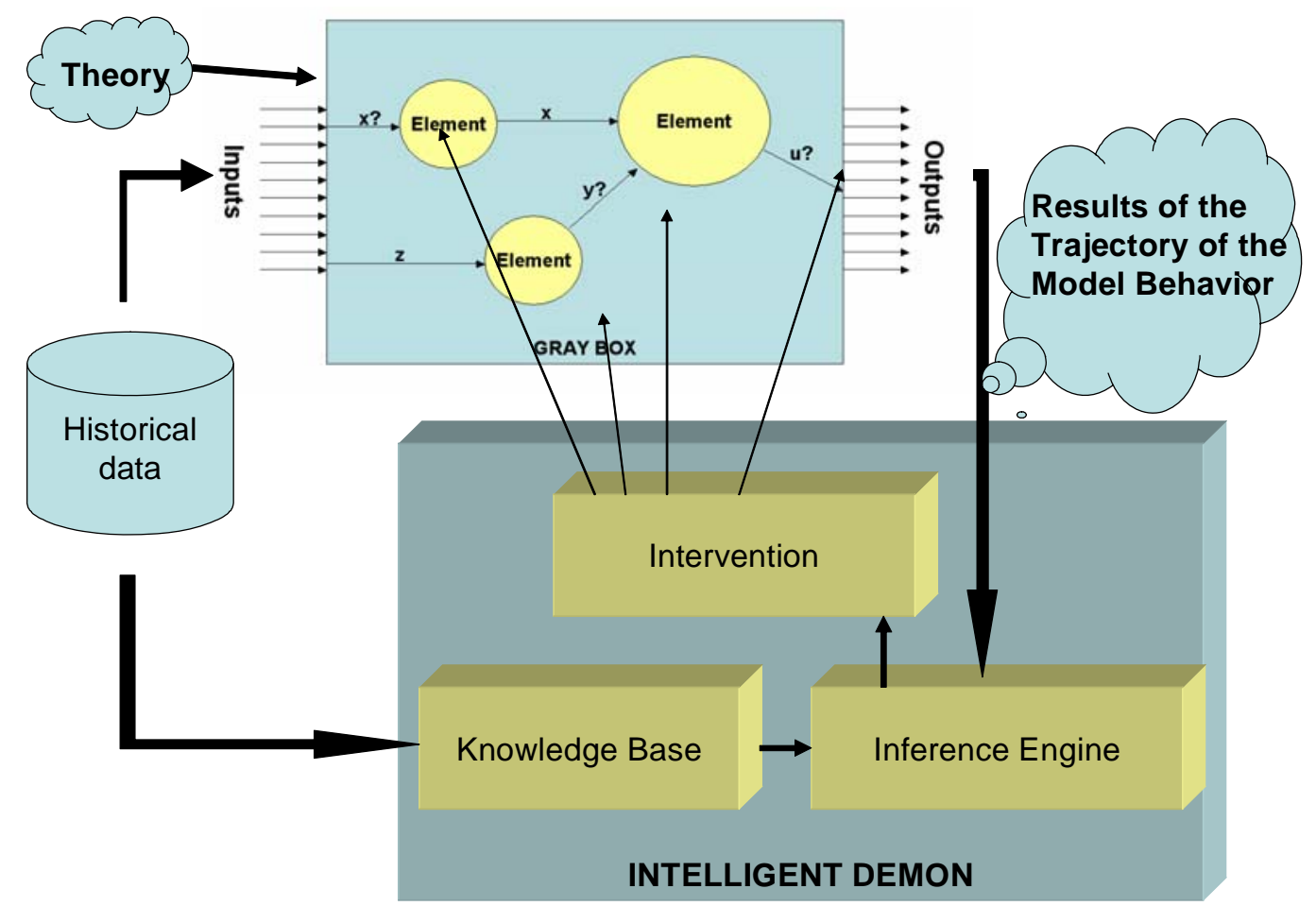

(Source: A. Jávor. "Demon Controlled Simulation”)

Fig. 3. Model identification by reconstruction

The same indicator can have very different meaning for different environmental elements: e.g. biodiversity is an irrelevant indicator for the artificial (built) environment directly, but it is very important from the point of view of living beings. Nevertheless air pollution has nearly the same importance for both elements: e.g. acid rains cause erosion on buildings and destroy forests as well.

As delineated it is not enough to choose the right indicators, but it is also needed to find and set the importance values (weights) for all possible elements. The following methodologies give the starting point for environmental assessment:

- A matrix $\mathbf{L}$ has to be constructed, where columns (indexed with $j$ ) give the environmental elements and rows (indexed with $i$ ) give the indicators themselves. Its elements $\mathrm{L}_{i j}$ determine a kind of correlation value, importance between rows and columns. (The matrix is analogous with Modified Leopold Matrix well known from the field of environmental impact assessment [7].)

- The matrix has to be normalized to one

- The relative values of matrix elements can be determined by AI controlled simulation

- Matrix elements should express importance deduced from direct influences on specific environmental element not considering secondary effects, these are viz. part of the dynamic simulation model symbolized by connections of Knowledge Attributed Petri Nets [6].
The main problem of importance of the indicators is how to determine values for non-human beings, when even human beings cannot determine their relation to several indicators. Therefore we suggest separating three types of importance:

- Known

- Anticipated

- Impacted (unknown).

Known type of importance is based on knowledge and real necessities. Anticipated importance is a self-determined thought qualifying that can have real and fake grounds. Impacted type importance is not directly known and can only be exposed by the methodology of model identification by reconstruction. The real importance value is compounded as the sum of the above mentioned importance.

After importance matrix had been defined it is possible to begin the evaluation of causes and effects. That can be carried out by AI controlled simulation (adequate simulation system is the CASSANDRA [5]). In that case causes and effects are mapped into another matrix $\mathbf{K}$ that is based on the Leopold Matrix and considers the following properties:

- Importance, cause and effect correlation (not equal to importance of indicators for environmental elements)

- Benefit

- Time of occurrence

- Duration 
Tab. 1. Importance of indicators (Modified Leopold Matrix)

\begin{tabular}{|c|c|c|c|c|c|c|c|}
\hline $\begin{array}{l}\text { Envirommental } \\
\text { elements } \rightarrow \\
\text { indicators } \downarrow\end{array}$ & flora & Fauna & Men & $\begin{array}{c}\text { Artifical } \\
\text { (built) } \\
\text { environment }\end{array}$ & $\begin{array}{l}\text { Non-living } \\
\text { natural } \\
\text { environment }\end{array}$ & Etc... & Sum(J) \\
\hline biodiversity & $L_{11}$ & $L_{12}$ & $L_{13}$ & $L_{14}$ & $L_{15}$ & $L_{1 j}$ & $\sum_{j} L_{1 j}$ \\
\hline air pollution & $L_{21}$ & $L_{22}$ & $L_{23}$ & $L_{24}$ & $L_{25}$ & $L_{2 j}$ & $\sum_{j} L_{2 j}$ \\
\hline water pollution & $L_{31}$ & $L_{32}$ & $L_{33}$ & $L_{34}$ & $L_{35}$ & $L_{3 j}$ & $\sum_{j} L_{3 j}$ \\
\hline soil pollution & $L_{41}$ & $L_{42}$ & $L_{43}$ & $L_{44}$ & $L_{45}$ & $L_{4 j}$ & $\sum_{j} L_{4 j}$ \\
\hline needed area & $L_{51}$ & $L_{52}$ & $L_{53}$ & $L_{54}$ & $L_{55}$ & $L_{5 j}$ & $\sum_{j} L_{5 j}$ \\
\hline state of buildings & $L_{61}$ & $L_{62}$ & $L_{63}$ & $L_{64}$ & $L_{65}$ & $L_{6 j}$ & $\sum_{j} L_{6 j}$ \\
\hline etc... & $L_{I 1}$ & $L_{I 2}$ & $L_{I 3}$ & $L_{I 4}$ & $L_{I 5}$ & $L_{I j}$ & $\sum_{j} L_{i j}$ \\
\hline $\operatorname{sum}(\mathrm{I})$ & $\sum_{i} L_{i 1}$ & $\sum_{i} L_{i 2}$ & $\sum_{i} L_{i 3}$ & $\sum_{i} L_{i 4}$ & $\sum_{i} L_{i 5}$ & $\sum_{i} L_{i j}$ & $\sum_{j} \sum_{i} L_{i j}$ \\
\hline
\end{tabular}

- Remedial measures

- Probability.

The mentioned properties (table entries) define a FUZZY like system. The sets can have the following names e.g. in case of Importance: MAJOR, MODERATE, MINOR, in case of Benefit: POSITIVE or NEGATIVE, or in case of Duration TRANSIENT, SHORT, PERMANENT, etc.

After all the matrix elements are functions, inference engines that have the above mentioned parameters. As known the prime Sugeno-type FUZZY relation has the following form [8, 9]:

$$
\begin{aligned}
& R_{i} \text { : } \\
& \quad \text { if } x_{i} \text { is } A_{1}^{i} \text { and } \ldots \text { and } x_{n} \text { is } A_{n}^{i} \\
& \text { then } y \text { is } c_{i 1}+\cdots+c_{i n} x_{n}+c_{i 0}, \quad i=1, \ldots, m
\end{aligned}
$$

According to the Leopold matrix relation between causes and effects can easily be expressed by their linear combination. Elements listed at causes can also be mentioned as effects and the other way round.

Therefore two matrices have to be constructed that are transposed in their headings, but have different elements.

As in Fig. 4 delineated elements of $\mathbf{K}$ matrix contain Wangtype FUZZY function approximations [9]; inference engines as linear combination of causes and effects. The entries are intelligent: they have influence on each other through parametric and topological connections. That concept can be mapped directly into Knowledge Attributed Petri Nets (KAPN) [6]. Global structural modification is possible through dynamic interventions of demons (intelligent agents) that execute model identification by reconstruction.

The methodology is detailed in the following:

- At start initializing the matrix elements (FUZZY decision systems) $\mathrm{K}_{i j}$ is needed

- The known parameters have to be built into the inference engines of entries

- Each cell has a state (FUZZY function parameters) and an output (normalized number between 0 and 1 to give appropriate numeric input to further model layers)
- The FUZZY model network as the state mentioned before (similar to Neuro-FUZZY systems [9]) have to be mapped into KAPN, where the places contain the $\mathrm{K}_{i j} \mathrm{FUZZY}$ coupling between cause and effect as knowledge attributes of the tokens

- Places of effects have coupling between each other according to secondary effects

- Tokens carry the information from one entry to the other

- Effects from incoming connections are additively taken into consideration

- Moving of tokens (mobile entities) change the marking of KAPN through the firing process, so transitions will dynamically determine which parameters in which combination will give the relevant impact on the FUZZY systems on the output side

- List of secondary or unsure effects have to be listed in the knowledge bases of demons to ensure model changeability and reconstruction

- After model had been initialized measuring of matrix output elements has to be carried out.

- Model identification by reconstruction have to be carried out to recognize the right system of couplings between environmental processes

- If the trajectory of model behaviour is not stable - as in most cases of environmental analysis - dynamic interventions have to be carried out, so impacted type of importance, detailed before, have to be raised or reduced

- When Modified Leopold Matrix (see Table ??) will change, importance of indicators will cause change in parameters of the environmental process (quality and quantity)

- In optimal case through demon controlled modification of entries in the two mentioned matrices and topology connected to KAPN based model can be reached a balanced stable state of the system. 


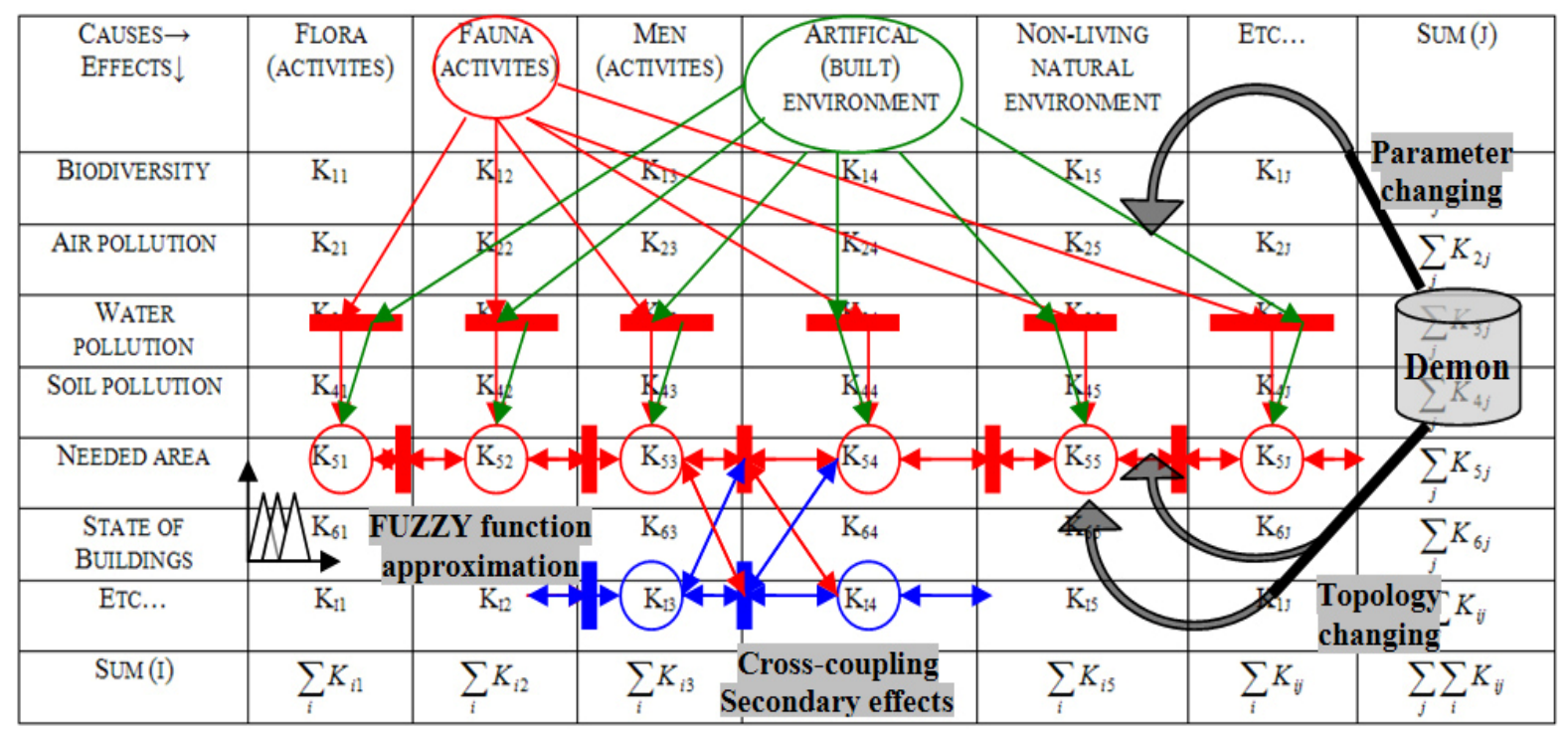

Fig. 4. Importance of indicators (Modified Leopold Matrix)

This methodology is appropriate to recognize unknown processes or to find secondary effects in environmental crosscoupled systems and is able to highlight the main problem of environmental indicators in out time, namely the anthropocentric approach. Using model identification by reconstruction it is possible to find new indicators that are more sensitive and can express better the need on intervention.

As main consequence it is delineated, that environmental assessment should not only be a static examination, but it is necessary to investigate the problem as a section at time $t_{n}$ of dynamic model behaviour.

Finally it is revealed that according to methodology, model synthesis is suggested to carry out from the fine structure to a robust one, therefore it is suited to plan local sustainability of regions.

\section{Spreading of environmental effects in physical space}

At environmental assessment processes there is another remarkable problem, namely the spreading effect of physical impacts in space and time. In most cases it is not possible to define crisp border-lines between physical spaces (e.g. regions), that are affected by the assessment. Local areas cannot be investigated separately considering environmental effects. To fulfil the requirements of trusted evaluation of environment trajectory of behaviour of neighbouring areas has to be taken into consideration: e.g. a locality with high level of air pollution indicates negative effects on other locality nearby. The consequences of this effect change dynamically fittingly to wind direction and strength. Other example could be the spreading of species: e.g. in time of today's high-graded agriculture impacts of monocultures, and biodiversity are relevant questions.

Causes and effects have pursuant to the above mentioned cellular behaviour with fuzzy couplings (see Fig. [5] [4].

The recognition of neighbourhood-effect bases the usage of CNN (Cellular Neural Network) description. In the following

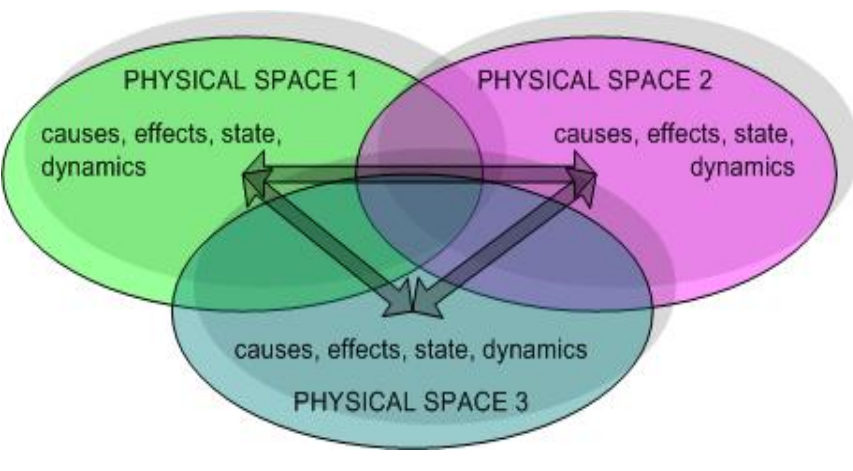

Fig. 5. Fuzzy couplings between cellular spaces

there is $\mathrm{CNN}$ methodology outlined that can describe changes of environmental properties of physical localities in space and time [3].

The CNN concept includes basically grids of elements in matrix form that have connections only to their neighbouring cells in a determined environment. There is a radius of the communication defined, that limits the complexity of the grid. If neighbouring radius is 1 , the connections have a weight in communication of 9 according to the number of neighbouring cells and the central cell. These weights are included into the template matrices.

$\mathrm{CNN}$ equations have in case of state-output the following form:

$$
\begin{aligned}
\dot{x}_{i j}(t) & =-x_{i j}(t)+\sum_{W_{r i j}^{x}} A_{k l} x_{i j}(t)+\sum_{W_{r i j}^{u}} B_{k l} u_{i j}+z_{i j} \\
x_{i j}^{\prime}(t) & =\frac{1}{2}\left(\left|x_{i j}(t)+1\right|\right)-\left(\left|x_{i j}(t)-1\right|\right)
\end{aligned}
$$

where $x_{i j}(t)$ is time-dependent (state-variable), $x_{i j}^{\prime}(t)$ is limited state, $u_{i j}$ is stands for input variable $z_{i j}$ is time-independent constant $\mathbf{A}$ and $\mathbf{B}$ are the template matrices Domains $W^{x} r_{i j}$ and $W^{u} r_{i j}$ express $\mathrm{r}$ radius environment of $x_{i j}$ and $u_{i j}$. Applying 
CNN methodology in EA can reveal new aspects that had been weighted insignificant before. This way of communication is not only suited to map the physical streaming of pollution, but is also appropriate to simulate the diffusion of effects in regions (See Figs. 6 and 7).
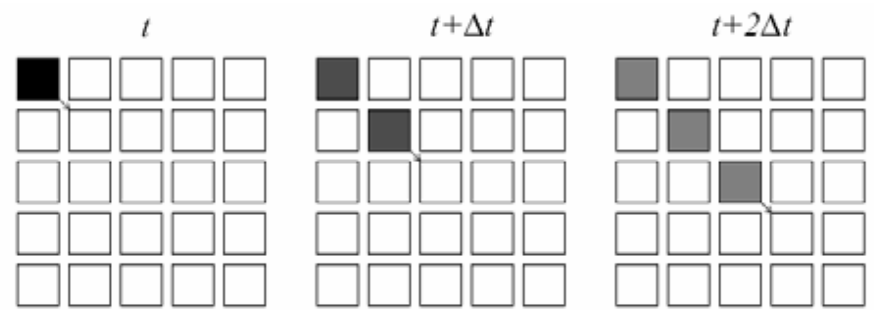

Fig. 6. Streaming of pollution from a high polluted area in south-east direction
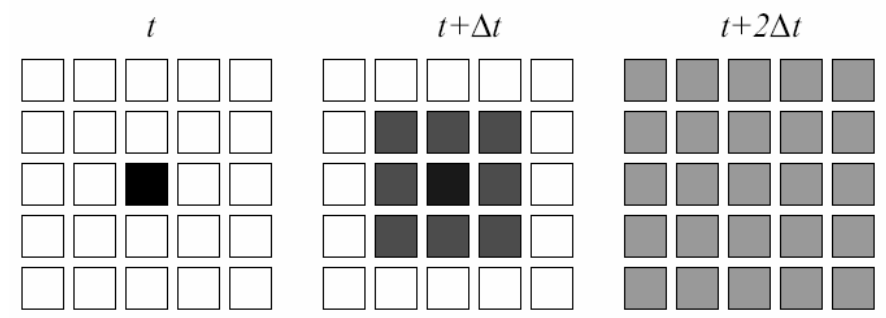

Fig. 7. Diffusion of effects

\section{Conclusion}

At discussion of the main requirements of environmental assessment can be mentioned the recognition of processes that cause changes in quality of environment, and explorations of causes and effects.

Information are needed that

- are relevant to the state of the environment at each point of time

- reveal the correlation between causes and effects

- denote the trend of changing.

This means, that composition of purposes concerning environmental policy is not possible without examining the state, changes in processes, and appropriate assessing. The main theoretical background should be the knowledge of social, economical and physical processes. According to this base the first and the most important step should be the environmental assessment that is suggested to carry out by alternative simulation methodologies outlined.

\section{References}

1 Marjai-Szerényi Zs, Economical Valuing Methodes in Environmental Mangement, 2005.

2 Szlávik J, Sustainable Environment- and Resourcemanagement, KJKKerszöv, 2005.

3 Bulla M, Methodological Development of Complex Environmental Assessment Systems, SZIE, Győr, 2004.
4 Für A, Tóth Á, Simulation of Nerve-Cell Network by Petri Nets, Alma Mater sorozat 9. (BME-GTK ITM), 2005.

5 Jávor A, Demon Controlled Simulation, Mathematics and Computers in Simulation 34 (1992), 283-296, DOI 10.1016/0378-4754(92)90006-3.

6 , Knowledge Attributed Petri Nets, Systems Analysis, Modelling, Simulation 13 (1993), no. 1/2, 5-12.

7 Barlow CA, Raisbeck JM, Lieberstein M, Varney RN, Water, Air \& Soil Pollution, Springer Netherlands, 2004.

8 Russel SJ, Norvig P, Artificial Intelligence: A Modern Approach, Prentice Hall, 2002.

9 Lantos B, Fuzzy Systems and Genetic Algorithms, Múegyetemi Kiadó, Budapest, 2002.

10 Jávor A, Simulation of Soft Systems, Informatics Bratislava, Slovak Republic, June 20 2005. Keynote lecture.

11 available at http://www.ceaa.gc.ca/010/basics_e.htm\{\#\}4

12 WorldBank Environmental Assessment SourceBook, 1999, available at http://siteresources.worldbank.org/ INTSAFEPOL/1142947-1118039018606/20532978/

Chapter4EconomicAnalysis0fProjectsAndPoliciesWith/ ConsiderationofEnvironmentalCostsAndBenefits.pdf 